\title{
Diophantine approximation on non-degenerate curves with non-monotonic error function
}

\author{
Natalia Budarina and Detta Dickinson
}

\begin{abstract}
It is shown that a non-degenerate curve in $\mathbb{R}^{n}$ satisfies a convergent Groshev theorem with a non-monotonic error function. In other words it is shown that if a volume sum converges the set of points lying on the curve which satisfy a Diophantine condition has Lebesgue measure zero.
\end{abstract}

\section{Introduction}

In 1924 Khintchine [13] proved that for almost all $x \in \mathbb{R}$ the inequality

$$
|q x-p|<\psi(q)
$$

has at most finitely or infinitely many solutions $p, q \in \mathbb{Z}$ according to the sum $\sum_{q=1}^{\infty} \psi(q)$ converges or diverges, where $\psi: \mathbb{R} \rightarrow \mathbb{R}^{+}$is a monotonic, decreasing function. This was extended to systems of linear forms by Groshev in 1938 [11]. For a polynomial $P \in \mathbb{Z}[x]$ let $H=H(P)$ denote the height of $P$; that is $H$ is the maximum of the modulus of the coefficients of $P$. Let $I \subset \mathbb{R}$ be an interval and define

$$
L_{n}(\psi)=\{x \in I:|P(x)|<\psi(H) \text { for infinitely many } P \in \mathbb{Z}[x], \operatorname{deg} P \leqslant n\} .
$$

Throughout, the Lebesgue measure of a measurable set $B \subset \mathbb{R}$ will be denoted by $\mu(B)$. In 1932 Mahler [15] showed that $\mu\left(L_{n}(\psi)\right)=0$ for $\psi(H)=H^{-w}$ if $w>4 n$ and conjectured the same result for any $w>n$. This conjecture was later proved by Sprindžuk [18]. In 1966 Baker [1] proved that $\mu\left(L_{n}(\psi)\right)=0$ if $\sum_{h=1}^{\infty} \psi^{1 / n}(h)$ converges and conjectured a stronger result, later known as Baker's conjecture [9]. This was finally proved in 1989 by Bernik [8] who showed that $\mu\left(L_{n}(\psi)\right)=0$ if the sum $\sum_{h=1}^{\infty} h^{n-1} \psi(h)$ converges. The divergence part was then proved by Beresnevich [2] in 1999 who showed that $\mu\left(L_{n}(\psi)\right)=|I|$ if $\sum_{h=1}^{\infty} h^{n-1} \psi(h)=\infty$. In all of the aforementioned results it was assumed that $\psi$ was monotonic.

Many of the results above have also been generalized to non-degenerate curves (that is, other than polynomials). Let $f_{1}, f_{2}, \ldots, f_{n}$ be $C^{n}$ functions from $I \rightarrow \mathbb{R}$ with Wronskian

$$
W(x)=\left|\begin{array}{ccc}
f_{1}^{\prime}(x) & \ldots & f_{n}^{\prime}(x) \\
\ldots & \ldots & \ldots \\
f_{1}^{(n)}(x) & \ldots & f_{n}^{(n)}(x)
\end{array}\right| \neq 0
$$

for almost all $x \in I$. When $n=2$ this is the same as saying that the curve $\left(f_{1}(x), f_{2}(x)\right)$ has non-zero curvature almost everywhere. In 1964 Schmidt [17] proved that such a planar curve is extremal. A curve $\Gamma=\left(f_{1}(x), \ldots, f_{n}(x)\right)$ is extremal on the interval $I$ if for almost all $x \in I$ the inequality

$$
\left|a_{n} f_{n}(x)+\ldots+a_{1} f_{1}(x)+a_{0}\right|<H^{-n-\varepsilon}
$$

Received 25 April 2008; revised 2 September 2008; published online 5 February 2009.

2000 Mathematics Subject Classification 11J83.

The first author is supported under the Science Foundation Ireland grant RFP05/MAT00015. 
has only a finite number of solutions $\mathbf{a}=\left(a_{0}, a_{1}, \ldots, a_{n}\right) \in \mathbb{Z}^{n+1}$ for all $\varepsilon>0$. In 1998, Kleinbock and Margulis [14] showed that every non-degenerate manifold in $\mathbb{R}^{n}$ is extremal. Analogues of the convergence case of the Khintchine and Groshev theorems were also obtained for non-degenerate manifolds independently by Beresnevich [3] and Bernik, Kleinbock and Margulis [10]. The divergence case was established in [6].

The convergence case of Khintchine's theorem holds without the condition that $\psi$ is monotonic. However, this is not true for the divergence case (see [12]). The question arises as to whether this condition is necessary in the case of convergence for manifolds. In 2005 Beresnevich [4] showed that the condition could be removed for the Veronese curve $G=\left(x, x^{2}, \ldots, x^{n}\right)$. In the same article he conjectured that the monotonicity condition could also be removed in the general situation of non-degenerate manifolds. In this paper we prove that his conjecture is correct for non-degenerate curves in Euclidean space.

\section{Main result}

Let $\mathcal{F}_{n}$ be the set of functions

$$
a_{n} f_{n}(x)+\ldots+a_{1} f_{1}(x)+a_{0},
$$

with $n \geqslant 2, \mathbf{a}=\left(a_{0}, \ldots, a_{n}\right) \in \mathbb{Z}^{n+1}$, and let $f_{1}, f_{2}, \ldots, f_{n}$ be $C^{n}$ functions from $I \rightarrow \mathbb{R}$ with non-vanishing Wronskian almost everywhere. For $F \in \mathcal{F}_{n}$ define the height of $F$ as $H=H(F)=\max _{1 \leqslant j \leqslant n}\left|a_{j}\right|$. Without loss of generality we will assume that $a_{n}=H$.

Throughout, the Vinogradov symbol $\ll$ is used so that if $K$ and $M$ are positive real numbers then $K \ll M$ means that there exists $C>0$ such that $K \leqslant C M$. If $K \ll M$ and $M \ll K$ then we write $K \asymp M$.

Our main result below is a convergent analogue of Groshev's theorem without monotonicity condition for the curve $\left\{\left(f_{1}(x), \ldots, f_{n}(x)\right): x \in \mathbb{R}\right\}$.

TheOREM 1. Let $\Psi: \mathbb{R} \rightarrow \mathbb{R}^{+}$be an arbitrary function (not necessarily monotonic) such that the sum $\sum_{h=1}^{\infty} h^{n-1} \Psi(h)$ converges. Let $L_{n}(\Psi)$ be the set of $x \in \mathbb{R}$ such that there are infinitely many $F \in \mathcal{F}_{n}$ satisfying

$$
|F(x)|<\Psi(H(F)) .
$$

Then $\mu\left(L_{n}(\Psi)\right)=0$.

\section{Preliminaries}

Since the sum $\sum_{h=1}^{\infty} h^{n-1} \Psi(h)$ converges then $H^{n-1} \Psi(H)$ tends to 0 as $H \rightarrow \infty$. Hence $H^{n-1} \Psi(H)=\mathrm{o}(1)$ and $\Psi(H)=\mathrm{o}\left(H^{-n+1}\right)$.

The set $S=\{x \in \mathbb{R}: W(x)=0\}$ is closed and of zero measure. Thus $\mathbb{R} \backslash S$ is open and, therefore, an $F_{\sigma}$ set. We can write $\mathbb{R} \backslash S=\cup_{k=1}^{\infty}\left[a_{k}, b_{k}\right]$. It is, therefore, sufficient to prove the theorem for a closed interval $I$. Also, since $|W(x)| \neq 0$ almost everywhere we will assume from now on, without loss of generality that

$$
|W(x)| \geqslant \varepsilon=\varepsilon(I)>0
$$

for all $x$ in such an interval $I$. We will also assume that there exists a constant $K_{0}$ such that for all $x \in I$

$$
\left|f_{j}(x)\right|<K_{0} \quad \text { and } \quad\left|f_{j}^{(i)}(x)\right|<K_{0}
$$

for $i, j=1, \ldots, n$. 
For the proof we will need some properties of the functions $F \in \mathcal{F}_{n}$. The following lemma is a modification and combination of Lemmas 2 and 3 of Pyartli [16]. We are assuming that (2) holds.

Lemma 1. Let $F \in \mathcal{F}_{n}$. For any interval $I_{1} \subset I$ with length $\left|I_{1}\right| \leqslant l=l\left(\varepsilon(I), K_{0}\right)$ there exists $i, 1 \leqslant i \leqslant n$, such that

$$
\left|F^{(i)}(x)\right|>c(l) H(F)
$$

for all $x \in I_{1}$.

The number of zeros of $F \in \mathcal{F}_{n}$ in $I_{1}$ does not exceed $n$.

Corollary 1. Let $F \in \mathcal{F}_{n}$. The number of sub-intervals in any interval $I_{1}$ with $\left|I_{1}\right| \leqslant$ $l\left(\varepsilon(I), K_{0}\right)$ where $F$ is monotonic is at most $n$.

Proof. Suppose that the number of intervals in $I_{1}$ where $F$ is monotonic is more than $n$. Then, the first derivative $F^{\prime}(x)$ has at least $n$ zeros in $I_{1}$. By Rolle's theorem, each derivative $F^{(j)}(x), 2 \leqslant j \leqslant n$, also has a zero in $I_{1}$. This contradicts Lemma 1.

Every interval $I$ can be written as a finite union of intervals $I_{1}$ with $\left|I_{1}\right| \leqslant l$. Therefore, it is sufficient to prove the theorem for each of these smaller intervals. From now on, we restrict ourselves to such an interval, relabelled $I$, which without loss of generality satisifies (4).

\section{Proof of Theorem}

To prove the theorem four different cases concerning the size of $\left|F^{\prime}(x)\right|$ are considered. If $x \in L_{n}(\psi)$ then $x$ must satisfy at least one of these cases infinitely often. To prove that each set of $x$ satisfying one of the conditions infinitely often has measure zero, repeated use will be made of the Borel-Cantelli Lemma below.

Lemma 2 Borel-Cantelli. Let $A_{j}$ be a family of Lebesgue measurable sets and let $A_{\infty}$ be the set of points $x \in \mathbb{R}$ which lie in infinitely many $A_{j}$. If $\sum_{j=1}^{\infty} \mu\left(A_{j}\right)<\infty$ then $\mu\left(A_{\infty}\right)=0$.

Case I. First the case of very small derivative is dealt with.

Lemma 3. The set of points $x \in I$ which satisfy

$$
\begin{aligned}
|F(x)| & <\Psi(H), \\
\left|F^{\prime}(x)\right| & <H^{-v},
\end{aligned}
$$

for infinitely many $F \in \mathcal{F}_{n}$ has measure zero.

This is proved using Theorem 1.4 from [10]. Using the notation in that theorem choose $T_{1}=\ldots=T_{n}=H, K=H^{-v}, \delta=H^{-n+1}$ and $l=n$, to obtain the following proposition.

Proposition 1. Let $I \subset \mathbb{R}$ be an interval and $\beta \in I$. Let $f=\left(f_{1}, \ldots, f_{n}\right)$ be an $n$-tuple of $C^{n}$ functions on $I$ which are non-degenerate at $\beta$. Then, there exists a finite interval $I_{0} \subset I$ 
containing $\beta$ and a constant $E>0$ such that

$$
\left|\bigcup_{F \in F_{n}, 0<H(F) \leqslant H}\left\{x \in I_{0}:|F(x)|<H^{-n+1},\left|F^{\prime}(x)\right|<H^{-v}\right\}\right| \leqslant E H^{-v /(n+1)(2 n-1)}\left|I_{0}\right| .
$$

\subsection{Proof of Lemma 3}

For a non-negative integer $k$ and for any $v>0$, we denote by $\mathcal{A}(k)$ the set of $x \in I_{0}$ such that the system of inequalities

$$
|F(x)| \ll H^{-n+1}, \quad\left|F^{\prime}(x)\right|<H^{-v}
$$

holds for some $F \in \mathcal{F}_{n}$ with $2^{k-1} \leqslant H(F)<2^{k}$. According to Proposition $1,|\mathcal{A}(k)| \ll$ $2^{-v k /(n+1)(2 n-1)}$ with $v>0$. The set of $x \in \mathbb{R}$ for which there are infinitely many $F \in \mathcal{F}_{n}$ satisfying (5) consists of points $x \in I_{0}$ which belong to infinitely many sets $\mathcal{A}(k)$. The sum $\sum_{k=1}^{\infty}|\mathcal{A}(k)|$ converges for $v>0$ and the Borel-Cantelli Lemma can be used to complete the proof of the lemma.

Thus from now on we may assume that $\left|F^{\prime}(x)\right|>H^{-v}$.

For the three remaining cases we need the following. The set of solutions of (1) in $I$ consists of at most $n$ intervals. Each of these intervals can be further divided into subintervals on which $F^{\prime}$ is also monotonic (at most $n-1$ of them). Each of these new intervals is finally further subdivided into intervals on which $F^{\prime}(x)$ is greater than or less than $H^{-v}$. Any interval on which $F^{\prime}(x)<H^{-v}$ has already been considered in Case I. For $F \in \mathcal{F}_{n}$, let $I_{j}(F)$ be one of the remaining intervals; thus, on $I_{j}(F), F$ and $F^{\prime}$ are monotonic and $F^{\prime}(x)>H^{-v}$ for all $x \in I_{j}(F)$. The number of $I_{j}(F)$ is clearly finite. Let $\bar{I}_{j}(F)$ denote the closure of $I_{j}(F)$ and let $\alpha_{j}$ denote a point in $\bar{I}_{j}(F)$ such that

$$
\left|F^{\prime}\left(\alpha_{j}\right)\right|=\min _{x \in \bar{I}_{j}(F)}\left|F^{\prime}(x)\right| .
$$

From the mean value theorem and (1), we obtain

$$
F(x)=F\left(\alpha_{j}\right)+F^{\prime}(\xi)\left(x-\alpha_{j}\right), \quad \xi \in\left[\alpha_{j}, x\right], x \in I_{j}(F) .
$$

From (1), we have

$$
\left|F(x)-F\left(\alpha_{j}\right)\right|<2 \Psi(H)
$$

Hence, if $F^{\prime}\left(\alpha_{j}\right) \neq 0$, then

$$
\left|x-\alpha_{j}\right|<2 \Psi(H)\left|F^{\prime}\left(\alpha_{j}\right)\right|^{-1}
$$

and $\mu\left(I_{j}(F)\right) \leqslant 2 \Psi(H)\left|F^{\prime}\left(\alpha_{j}\right)\right|^{-1}$.

Let $\delta>0$ be a fixed real number. As $\delta \rightarrow 0$ the measure of the set of $x \in I$ for which the inequality $\left|f_{s}(x)\right| \leqslant \delta$ holds for at least one $s, 1 \leqslant s \leqslant n$, also tends to zero. Hence, from now on it is assumed that

$$
\left|f_{i}(x)\right|>\delta, \quad 1 \leqslant i \leqslant n .
$$

In what follows define the function $t_{i j}$ as $t_{i j}(x)=f_{i}(x) f_{j}^{-1}(x)$. It is shown in [5, Lemma 3] that if $W(x) \neq 0$ almost everywhere then $t_{i j}^{\prime}(x) \neq 0$ almost everywhere for all $i, j \in\{1, \ldots, n\}$. The next lemma relates the size of $|W(x)|$ to the size of $\left|f_{i}(x) f_{j}^{\prime}(x)-f_{i}^{\prime}(x) f_{j}(x)\right|$.

LEMma 4. If $|W(x)| \geqslant \varepsilon$ then $\left|f_{i}(x) f_{j}^{\prime}(x)-f_{i}^{\prime}(x) f_{j}(x)\right|>\left(\varepsilon \delta^{2}\right) /\left(2^{n+1} n ! K_{0}^{n}\right)$ for all $i, j$ in $\{1, \ldots, n\}$. 
Proof. Let $\delta_{1}>0$ be a constant (to be chosen later) and assume that $\left|t_{i j}^{\prime}(x)\right| \leqslant \delta_{1}$. Then, from

$$
\left|t_{i j}^{\prime}(x)\right|=\left|\frac{f_{i}^{\prime}(x) f_{j}(x)-f_{i}(x) f_{j}^{\prime}(x)}{f_{j}^{2}(x)}\right| \leqslant \delta_{1},
$$

we obtain

$$
f_{i}^{\prime}(x)=f_{j}^{\prime}(x) \frac{f_{i}(x)}{f_{j}(x)}+\theta(x) f_{j}(x) \delta_{1},
$$

where $|\theta(x)| \leqslant 1$. This can be rewritten as

$$
f_{i}^{\prime}(x)=f_{j}^{\prime}(x) \frac{f_{i}(x)}{f_{j}(x)}+\delta_{1} u_{1}(x)
$$

where $u$ is a bounded $(n-1)$-times continuously differentiable function (note that $\left|f_{j}(x)\right|>\delta$ ). Differentiate the above equation and use (8) to obtain

$$
f_{i}^{\prime \prime}(x)=f_{j}^{\prime \prime}(x) \frac{f_{i}(x)}{f_{j}(x)}+f_{j}^{\prime}(x) t_{i j}^{\prime}(x)+\delta_{1} u_{1}^{\prime}(x)=f_{j}^{\prime \prime}(x) \frac{f_{i}(x)}{f_{j}(x)}+\delta_{1} u_{2}(x) .
$$

Further differentiating with respect to $x$, for $2 \leqslant k \leqslant n-1$ gives

$$
f_{i}^{(k+1)}(x)=f_{j}^{(k+1)}(x) \frac{f_{i}(x)}{f_{j}(x)}+\delta_{1} u_{k+1}(x) .
$$

Replace column $i$ in $W(x)$ with (9)-(11) and write it as

$$
W(x)=\frac{f_{i}(x)}{f_{j}(x)} W_{1}(x)+\delta_{1} W_{2}(x) .
$$

In $W_{1}(x)$ the $i$ th and $j$ th columns are equal so that $\left|W_{1}(x)\right| \equiv 0$, and from (3) it is readily verified that $\left|W_{2}\right|<2^{n} n ! K_{0}^{n}$. Hence, $|W(x)|<2^{n} \delta_{1} n ! K_{0}^{n}$. For $\delta_{1}<\varepsilon /\left(2^{n} n ! K_{0}^{n}\right)$ this contradicts $|W(x)| \geqslant \varepsilon$. Thus, from $(7),\left|f_{i}(x) f_{j}^{\prime}(x)-f_{i}^{\prime}(x) f_{j}(x)\right|>\varepsilon \delta^{2} /\left(2^{n+1} n ! K_{0}^{n}\right)$.

From now on, it is, therefore, assumed without loss of generality that

$$
\left|f_{i}(x) f_{j}^{\prime}(x)-f_{i}^{\prime}(x) f_{j}(x)\right| \geqslant \delta_{2}=\frac{\varepsilon \delta^{2}}{2^{n+1} n ! K_{0}^{n}}
$$

for all $i, j \in\{1, \ldots, n\}$ with $i \neq j$.

Lemma 5. Let $I \subset \mathbb{R}$ be an interval for which $|W(x)| \geqslant \varepsilon$. Let $B_{1} \subset \mathbb{R}$ be a set with $\mu\left(B_{1}\right)=0$ and let $B_{2}=\left\{x \in I: t_{i j}(x) \in B_{1}\right\}$, then $B_{2}$ also has zero measure.

Proof. As $|W(x)| \geqslant \varepsilon$, it follows from Lemma 4 that $\left|t_{i j}^{\prime}(x)\right| \geqslant \delta_{1}$ for all $x \in I$. Hence, if $E \subset I$ is an interval then $\left.\delta^{2} \mu\left(t_{i j}(E)\right) /\left(2 K_{0}^{2}\right) \leqslant \mu(E)\right) \leqslant \mu\left(t_{i j}(E)\right) / \delta_{1}$ (using the mean value theorem). If $\mu\left(B_{1}\right)=0$ then for each $\eta>0$ there exists a countable collection of intervals $I_{i}$ such that $B_{1} \subset \cup_{i=1}^{\infty} I_{i}$ with $\sum_{i=1}^{\infty} \mu\left(I_{i}\right)<\eta$. Let $J_{i}$ be such that $t_{i j}\left(J_{i}\right)=I_{i}$. Since $t_{i j}^{\prime}(x) \neq 0$ for all $x \in I, J_{i}$ must also be an interval and

$$
\frac{\delta^{2} \mu\left(I_{i}\right)}{2 K_{0}^{2}} \leqslant \mu\left(J_{i}\right) \leqslant \frac{\mu\left(I_{i}\right)}{\delta_{1}} .
$$

Clearly $B_{2} \subset \cup_{i=1}^{\infty} J_{i}$ which implies that $B_{2}$ must also have measure zero.

Now we are ready to complete the proof of Theorem 1 . The three remaining cases in the proof concern different ranges for the size of $F^{\prime}\left(\alpha_{j}\right)$. 
Case II. For $F \in \mathcal{F}_{n}$, let $\sigma(F)$ be the union of intervals $I_{j}(F)$ for which $\left|F^{\prime}\left(\alpha_{j}\right)\right| \geqslant c_{1} H^{1 / 2}$. Hence, $\sigma(F)$ is the set of $x \in I$ which satisfy

$$
|F(x)|<\Psi(H),
$$

so that $x$ lies in some interval $I_{j}(F)$ and

$$
\left|F^{\prime}\left(\alpha_{j}\right)\right| \geqslant c_{1} H^{1 / 2} .
$$

For a constant $c_{2}=c_{2}(n)$ define the set $\sigma_{1}(F)$ of points $x$ which satisfy

$$
\left|x-\alpha_{j}\right|<c_{2}\left|F^{\prime}\left(\alpha_{j}\right)\right|^{-1}
$$

for $\alpha_{j} \in \sigma(F)$.

From (6), for $H>H_{0}\left(c_{2}\right)$, the inequality $\sigma(F) \subset \sigma_{1}(F)$ holds and

$$
\mu(\sigma(F))<2 c_{2}^{-1} \Psi(H) \mu\left(\sigma_{1}(F)\right) .
$$

For each $j$ with $\alpha_{j} \in \sigma(F)$ develop $F$ as a Taylor series on $\sigma_{1}(F)$, so that

$$
F(x)=F\left(\alpha_{j}\right)+F^{\prime}\left(\alpha_{j}\right)\left(x-\alpha_{j}\right)+\frac{F^{\prime \prime}\left(\xi_{1}\right)\left(x-\alpha_{j}\right)^{2}}{2}, \quad \xi_{1} \in\left[\alpha_{j}, x\right] .
$$

Estimate each term in the above equation to obtain

$$
\begin{aligned}
\left|F\left(\alpha_{j}\right)\right| & <\Psi(H)<c_{2}, \\
\left|F^{\prime}\left(\alpha_{j}\right)\left(x-\alpha_{j}\right)\right| & <c_{2}, \\
\left|F^{\prime \prime}\left(\alpha_{j}\right)\left(x-\alpha_{j}\right)^{2}\right| & <c_{3} H\left(c_{2}\left|F^{\prime}\left(\alpha_{j}\right)\right|^{-1}\right)^{2}=c_{3} c_{2}^{2} c_{1}^{-2} .
\end{aligned}
$$

It is possible to choose $c_{2}=c_{2}(\delta)<\delta / 10$ such that $c_{3} c_{2} c_{1}^{-2}<1$. Thus, from (13) $|F(x)|<3 c_{2}$ for $H>H_{0}\left(c_{2}\right)$.

Fix the vector $\mathbf{b}=\left(H, a_{n-1} \ldots, a_{2}, a_{0}\right)$, and let the subclass $\mathcal{F}_{n}$ of functions with the same vector $\mathbf{b}$ be denoted by $\mathcal{F}_{n}(\mathbf{b})$. The number of different $\mathcal{F}_{n}(\mathbf{b})$ is $\ll H^{n-1}$. Let $F_{1}, F_{2} \in \mathcal{F}_{n}(\mathbf{b})$, and assume that they have different coefficients $a_{1}$. Also, assume that $\sigma_{1}\left(F_{1}\right) \cap \sigma_{1}\left(F_{2}\right) \neq \emptyset$, for $F_{j} \in \mathcal{F}_{n}(\mathbf{b}), j=1,2$. Let $R(x)=F_{2}(x)-F_{1}(x)=a_{1}\left(F_{2}\right) f_{1}(x)-a_{1}\left(F_{1}\right) f_{1}(x)=a_{1}^{\prime} f_{1}(x)$, where $\left|a_{1}^{\prime} f_{1}(x)\right|>\delta$. Here, $a_{i}\left(F_{j}\right)$ denotes the $i$ th coordinate of $F_{j}$. Then,

$$
\delta<|R(x)| \leqslant 6 c_{2}<\frac{3 \delta}{5}
$$

which is a contradiction. Hence, $\sigma_{1}\left(F_{1}\right) \bigcap \sigma_{1}\left(F_{2}\right)=\emptyset$ and

$$
\sum_{F \in \mathcal{F}_{n}(\mathbf{b})} \mu\left(\sigma_{1}(F)\right) \ll|I|
$$

Together with (14) this gives

$$
\sum_{F \in \mathcal{F}_{n}(\mathbf{b})} \mu(\sigma(F)) \ll|I| \Psi(H) .
$$

Summing this over all vectors $\mathbf{b}$ gives

$$
\sum_{H=1}^{\infty} \sum_{\mathbf{b}} \sum_{F \in \mathcal{F}_{n}(\mathbf{b})} \mu(\sigma(F)) \ll \sum_{H=1}^{\infty} H^{n-1} \Psi(H)|I|<\infty .
$$

The Borel-Cantelli lemma can now be used to complete the proof.

Case III. This time, use $\sigma(F)$ to denote the union of intervals $I_{j}(F)$ for which $1 \leqslant\left|F^{\prime}\left(\alpha_{j}\right)\right|<$ $c_{1} H^{1 / 2}$. Hence $\sigma(F)$ is the set of $x \in I$ which satisfy

$$
|F(x)|<\Psi(H)
$$


so that $x$ lies in some $I_{j}(F)$ and

$$
1 \leqslant\left|F^{\prime}\left(\alpha_{j}\right)\right|<c_{1} H^{1 / 2}
$$

First assume that $n>2$; the case $n=2$ will be dealt with at the end. Define the set $\sigma_{2}(F) \supset \sigma(F)$ as the set of points $x$ which satisfy the inequality

$$
\left|x-\alpha_{j}\right|<c_{4} H^{-1}\left|F^{\prime}\left(\alpha_{j}\right)\right|^{-1}
$$

for $\alpha_{j} \in \sigma(F)$. It is clear that

$$
\mu(\sigma(F))<2 c_{4}^{-1} H \Psi(H) \mu\left(\sigma_{2}(F)\right) .
$$

Fix the vector $\mathbf{b}_{1}=\left(H, a_{n-1} \ldots, a_{3}, a_{0}\right)$ and denote the subclass $\mathcal{F}_{n}$ of functions with the same vector $\mathbf{b}_{1}$ by $\mathcal{F}_{n}\left(\mathbf{b}_{1}\right)$. The number of different sets $\mathcal{F}_{n}\left(\mathbf{b}_{1}\right)$ is $O\left(H^{n-2}\right)$. The domain $\sigma_{2}(F)$ is called inessential if there is a function $\tilde{F} \in \mathcal{F}_{n}\left(\mathbf{b}_{1}\right)$ (with $\tilde{F} \neq F$ ) such that

$$
\mu\left(\sigma_{2}(F) \bigcap \sigma_{2}(\tilde{F})\right) \geqslant \frac{\mu\left(\sigma_{2}(F)\right)}{2},
$$

and essential otherwise.

First, the essential domains are investigated. By definition

$$
\sum_{F \in \mathcal{F}_{n}\left(\mathbf{b}_{1}\right)} \mu\left(\sigma_{2}(F)\right) \ll|I| .
$$

From this, (16) and the fact that the number of vectors $\mathbf{b}_{1}$ is $O\left(H^{n-2}\right)$, we have

$$
\sum_{\mathbf{b}_{1}} \sum_{F \in \mathcal{F}_{n}\left(\mathbf{b}_{1}\right)} \mu(\sigma(F)) \ll H^{n-1} \Psi(H)|I| .
$$

Finally, we obtain

$$
\sum_{H=1}^{\infty} \sum_{\mathbf{b}_{1}} \sum_{F \in \mathcal{F}_{n}\left(\mathbf{b}_{1}\right)} \mu(\sigma(F))<\infty
$$

Thus, by the Borel-Cantelli Lemma, the set of points $x$ which belong to infinitely many essential domains is of measure zero.

Now we consider the inessential domains. Develop every function $F \in \mathcal{F}_{n}\left(\mathbf{b}_{1}\right)$ as a Taylor series on the interval $\sigma_{2}(F)$ so that

$$
F(x)=F\left(\alpha_{j}\right)+F^{\prime}\left(\alpha_{j}\right)\left(x-\alpha_{j}\right)+\frac{F^{\prime \prime}\left(\xi_{2}\right)\left(x-\alpha_{j}\right)^{2}}{2}, \quad \xi_{2} \in\left[\alpha_{j}, x\right], \quad \alpha_{j} \in \sigma(F),
$$

and estimate each term of the decomposition from above to obtain

$$
\begin{aligned}
\left|F\left(\alpha_{j}\right)\right| & <c_{5} H^{-1} \\
\left|F^{\prime}\left(\alpha_{j}\right)\left(x-\alpha_{j}\right)\right| & <c_{5} H^{-1} \\
\left|F^{\prime \prime}\left(\xi_{2}\right)\left(x-\alpha_{j}\right)^{2}\right| & <c_{5} H H^{-2}\left|F^{\prime}\left(\alpha_{j}\right)\right|^{-2}<c_{5} H H^{-2} 1=c_{5} H^{-1}
\end{aligned}
$$

for $\alpha_{j} \in \sigma(F)$ and some constant $c_{5}>0$; the last estimate comes from (15). We conclude that

$$
|F(x)|<3 c_{5} H^{-1} .
$$

Furthermore, from the mean value theorem, for $x \in \sigma_{2}(F)$ with $\alpha_{j} \in \sigma(F)$,

$$
\begin{aligned}
\left|F^{\prime}(x)\right| & \leqslant\left|F^{\prime}\left(\alpha_{j}\right)\right|+\left|F^{\prime \prime}(\xi)\left(x-\alpha_{j}\right)\right| \\
& \ll H^{1 / 2}+H H^{-1}\left|F^{\prime}\left(\alpha_{j}\right)\right|^{-1} \ll H^{1 / 2} .
\end{aligned}
$$

Consider the new function $R=F_{2}-F_{1}=a_{1}^{\prime} f_{1}+a_{2}^{\prime} f_{2}$, where both $F_{1}$ and $F_{2}$ belong to $\mathcal{F}_{n}\left(\mathbf{b}_{1}\right)$. For these functions, conditions (17) and (18) hold on the set $\sigma_{2}\left(F_{1}\right) \bigcap \sigma_{2}\left(F_{2}\right)$. By $(7),(17)$ and 
(18), we obtain

$$
|R(x)| \ll H^{-1}, \quad\left|R^{\prime}(x)\right| \ll H^{1 / 2} .
$$

From (12) it is relatively straightforward to show that $\left|a_{i}^{\prime}\right| \ll H^{1 / 2}$ for $i=1,2$ so that $H(R)<c_{7} H^{1 / 2}$. Therefore, $\left|a_{1}^{\prime} f_{1}(x)+a_{2}^{\prime} f_{2}(x)\right| \ll H(R)^{-2}$. Divide by $f_{1}(x)$ and set $t=t_{21}=$ $f_{2} f_{1}^{-1}$. Then, $\left|a_{2}^{\prime} t(x)+a_{1}^{\prime}\right| \ll H(R)^{-2}$ which, by Khintchine's Theorem, holds infinitely often only on a set of measure zero. Finally, by Lemma 5 , the set of points $x \in I$ which satisfy $|R(x)| \ll H^{-1}$ for infinitely many $\left(a_{1}^{\prime}, a_{2}^{\prime}\right)$ also has zero measure.

Lastly, we consider the case $n=2$. The proof follows the same lines as above except that instead of restricting to the sets $\mathcal{F}_{n}\left(\mathbf{b}_{1}\right)$ we restrict to the set $\mathcal{F}_{n}(H)$, which is the set of $F \in \mathcal{F}_{n}$ with height $H$. For the essential intervals the proof is exactly the same and we obtain

$$
\sum_{H=1}^{\infty} \sum_{F \in \mathcal{F}_{n}(H)} \mu(\sigma(F)) \ll \sum_{H=1}^{\infty} H \Psi(H)|I|<\infty .
$$

For the inessential intervals the proof is exactly the same up to equation (18). Then, $R(x)=$ $F_{2}(x)-F_{1}(x)=a f_{1}(x)+b$ for some constants $a, b \ll H$. Then, as before $|R(x)| \ll H^{-1}$ and $\left|R^{\prime}(x)\right|=\left|a f_{1}^{\prime}(x)\right| \ll H^{1 / 2}$. Since $\left|f_{1}^{\prime}(x)\right|<K_{0}$ this implies that $a \ll H^{1 / 2}$ and the proof is completed as before.

Case $I V$. This is very similar to the previous case. Use $\sigma(F)$ to denote the union of intervals $I_{j}(F)$ for which $H^{-v} \leqslant\left|F^{\prime}\left(\alpha_{j}\right)\right|<1$ with $0<v<1 / 3$. Hence $\sigma(F)$ is the set of $x \in I$ which satisfy

$$
|F(x)|<\Psi(H)
$$

so that $x$ lies in some $I_{j}(F)$ and

$$
H^{-v} \leqslant\left|F^{\prime}\left(\alpha_{j}\right)\right|<1
$$

Treat the case $n=2$ exactly as in Case III. For $n>2$ fix the vector $\mathbf{b}_{1}$ as above and define the domains:

$$
\begin{aligned}
\sigma(F) & :\left|x-\alpha_{j}\right|<2 \Psi(H)\left|F^{\prime}\left(\alpha_{j}\right)\right|^{-1} \\
\sigma_{3}(F) & :\left|x-\alpha_{j}\right|<c_{8} H^{-1}\left|F^{\prime}\left(\alpha_{j}\right)\right|^{-1} .
\end{aligned}
$$

From this,

$$
\mu(\sigma(F)) \leqslant 2 c_{8}^{-1} \mu\left(\sigma_{3}(F)\right) H \Psi(H) .
$$

As in Case III we use essential and inessential domains. Summing the measures of the essential domains $\sigma_{3}(F)$ gives

$$
\sum_{F \in \mathcal{F}_{n}\left(\mathbf{b}_{1}\right)} \mu\left(\sigma_{3}(F)\right) \ll|I|
$$

As $\# \mathbf{b}_{1} \ll H^{n-2}$, from (19) and (20), we have

$$
\sum_{H=1}^{\infty} \mu(\sigma(F)) \ll \sum_{H=1}^{\infty} H^{n-1} \Psi(H)|I|<\infty .
$$

By the Borel-Cantelli Lemma, the set of those $x$ belonging to infinitely many essential domains $\sigma(F)$ has zero measure. 
Now let $\sigma_{3}(F)$ be an inessential domain. Using Taylor's formula for $F$ on $\sigma_{3}(F)$, we obtain

$$
\begin{aligned}
F(x) & =F\left(\alpha_{j}\right)+F^{\prime}\left(\alpha_{j}\right)\left(x-\alpha_{j}\right)+\frac{1}{2} F^{\prime \prime}\left(\xi_{3}\right)\left(x-\alpha_{j}\right)^{2}, \quad \xi_{3} \in\left[\alpha_{j}, x\right], \\
\left|F\left(\alpha_{j}\right)\right| & <\Psi(H)<c_{9} H^{-1}, \\
\left|F^{\prime}\left(\alpha_{j}\right)\left(x-\alpha_{j}\right)\right| & <c_{9} H^{-1}, \\
\left|F^{\prime \prime}\left(\xi_{3}\right)\left(x-\alpha_{j}\right)^{2}\right| & <c_{9} H H^{-2}\left|F^{\prime}\left(\alpha_{j}\right)\right|^{-2}<c_{9} H^{2 v-1},
\end{aligned}
$$

and finally,

$$
|F(x)|<3 c_{9} H^{2 v-1} .
$$

By the mean value theorem, for any $x \in \sigma_{3}(F)$

$$
\begin{aligned}
\left|F^{\prime}(x)\right| & \leqslant\left|F^{\prime}\left(\alpha_{j}\right)\right|+\left|F^{\prime \prime}(\xi)\left(x-\alpha_{j}\right)\right| \\
& \ll 1+H H^{-1}\left|F^{\prime}\left(\alpha_{j}\right)\right|^{-1} \ll H^{v} .
\end{aligned}
$$

Consider $R(x)=F_{2}(x)-F_{1}(x)$ with $F_{1}, F_{2} \in \mathcal{F}_{n}\left(\mathbf{b}_{1}\right)$ and $x \in \sigma_{3}\left(F_{1}\right) \bigcap \sigma_{3}\left(F_{2}\right)$. For $R$ the inequalities $|R(x)|<c_{10} H^{2 v-1}$ and $\left|R^{\prime}(x)\right|<c_{11} H^{v}$ hold; these follow from (21) and (22). As in Case III it is possible to show from (12) that $\left|a_{i}^{\prime}\right| \ll H^{v} \quad(i=1,2)$ so that $H(R)=\max \left\{\left|a_{1}^{\prime}\right|,\left|a_{2}^{\prime}\right|\right\} \ll H^{v}$. Again, let $t=t_{21}=f_{2} f_{1}^{-1}$. By $(7)$ and $(21),|R(x)|=\mid a_{2}^{\prime} t(x)+$ $a_{1}^{\prime} \mid<c_{10} \delta^{-1} H^{2 v-1} \ll H(R)^{(2 v-1) / v}$ for $v<1 / 3$. By Khintchine's theorem the last inequality holds infinitely often only for a set of measure zero. Hence, by Lemma 5 , the measure of $\sigma_{3}\left(F_{1}\right) \bigcap \sigma_{3}\left(F_{2}\right)$ is zero and the measure of the set of $x$ which belong to infinitely many inessential domains is also zero. The proof of the theorem is therefore complete.

In this paper only the case of non-degenerate curves has been proved. This leaves the remaining question of non-degenerate manifolds and a non-monotonic error function. We are currently considering this question in some ongoing work. There are also some recent results in the theory of simultaneous (rather than dual) Diophantine approximation on manifolds; in particular, a divergent Khintchine theorem [7] and a corresponding convergent Khintchine Theorem [19] have been obtained. In both of these articles the error function is required to be monotonic. Thus the same question can be asked in simultaneous approximation on manifolds: can the monotonicity condition be removed?

Acknowledgements. We would like to thank Basil Bernik for drawing our attention to this problem and as always for his very helpful comments. We are also grateful to an anonymous referee for some extremely astute comments.

\section{References}

1. A. Baker, 'On a theorem of Sprindžuk', Proc. R. Soc. London Ser. A 292 (1966) 92-104.

2. V. V. Beresnevich, 'On approximation of real numbers by real algebraic numbers', Acta Arith. 90 (1999) $97-112$.

3. V. V. Beresnevich, 'A Groshev type theorem for convergence on manifolds', Acta Math. Hungar. 94 (2002) 99-130.

4. V. V. Beresnevich, 'On a theorem of V. Bernik in the metric theory of Diophantine approximation', Acta Arith. 1171 (2005) 71-80.

5. V. V. Beresnevich and V. I. Bernik, 'On a metrical theorem of W. Schmidt', Acta Arith. 75 (1996) 219-233.

6. V. V. Beresnevich, V. I. Bernik, D. Y. Kleinbock and G. A. Margulis, 'Metric Diophantine approximation: the Khintchine-Groshev theorem for non-degenerate manifolds', Moscow Math. J. 2 (2002) 203-225.

7. V. V. Beresnevich, D. Dickinson and S. L. Velani, 'Diophantine approximation on planar curves and the distribution of rational points', (with an appendix by R. C. Vaughn), Ann. Math. 166 (2007) 367-426.

8. V. I. Bernik, 'On the exact order of approximation of zero by values of integral polynomials', Acta Arith. 53 (1989) 17-28.

9. V. I. Bernik and M. M. Dodson, Metric Diophantine approximation on manifolds, Cambridge Tracts in Mathematics 137 (CUP, Cambridge, 1999). 
10. V. I. Bernik, D. Y. Kleinbock and G. A. Margulis, 'Khintchine-type theorems on manifolds: the convergence case for standard and multiplicative versions', Internat. Math. Res. Notices 9 (2001) 453-486.

11. A. V. Groshev, Une théorème sur les systèmes des forme linéares, Doklady Akademii Nauk SSSR 1938, 151-152.

12. G. Harman, Metric number theory, LMS Monographs New Series 18 (Clarendon Press, Oxford, 1998).

13. A. Khintchine, 'Einige Sätze über Kettenbrüche mit Anwendungen auf die Theorie der Diophantischen Approximationen', Math. Ann. 92 (1924) 115-125.

14. D. Y. Kleinbock and G. A. MARgulis, 'Flows on homogeneous spaces and Diophantine approximation on manifolds', Ann. Math. 148 (1998) 339-360.

15. K. Mahler, 'Über das Mass der Menge aller S-Zahlen', Math. Ann. 106 (1932) 131-139.

16. A. S. Pyartli, 'Diophantine approximations on submanifolds of Euclidean space', Funct. Anal. Appl. 3 (1970) 303-306.

17. W. Schмidt, 'Metrische Sätze über simultane Approximation abhängiger Grössen', Monatsh. Math. 63 (1964) 154-166.

18. V. SPRINDŽUK, Mahler's problem in the metric theory of numbers, Translations of Mathematical Monographs 25 (American Mathematical Society, Providence, RI, 1969).

19. S. L. Velani and R. C. Vaughn, 'Diophantine approximation on planar curves: the convergence theory', Invent. Math. 166 (2006) 103-124.

\section{Natalia Budarina and Detta Dickinson}

Department of Mathematics

Logic House

National University of Ireland, Maynooth

Co Kildare

Republic of Ireland

nbudarina@maths.nuim.ie

ddickinson@maths.nuim.ie 